\title{
Teilnehmer des Symposions
}

Prof. Dr. Dr. h.c. Okko Behrends, Göttingen

Prof. Dr. Uwe Diederichsen, Göttingen

Prof. Dr. Dr. h.c. Eberhard Eichenhofer, Jena

Prof. DDr. Christoph Grabenwarter, Wien

Prof. Dr. Dr. h.c. Werner Heun, Göttingen

Prof. Dr. Christian Hillgruber, Bonn

Prof. Dr. Eva-Maria Kieninger, Würzburg

Prof. Dr. Dr. h.c. mult. Christoph Link, Erlangen

Prof. Dr. Peter Oestmann, Münster

Prof. Dr. Andreas Paulus, Göttingen

Dr. Enrico Peuker, Jena

Prof. Dr. Matthias Ruffert, Jena

Prof. Dr. Christoph Schönberger, Konstanz

Prof. Dr. Frank Schorkopf, Göttingen

Prof. Dr. Eva Schumann, Göttingen

Prof. Dr. Wolfgang Sellert, Göttingen

Prof. Dr. Christian Starck, Göttingen

Prof. Dr. Michael Stürner, M. Jur. (Oxford), Konstanz

PD Dr. Alexander Thiele, Göttingen

Prof. Dr. Christiane Wendehorst, LL. M., Wien

Prof. Dr. Dr. h.c. mult. Reinhard Zimmermann, Hamburg

Die Betreuung des Tagungsbandes oblag am Institut Herrn Wiss. Mitarbeiter Carsten Fitting, dem die Herausgeberin ebenso wie allen anderen Mitarbeitern und Hilfskräften, die von der Organisation der Tagung bis zur Drucklegung des Tagungsbandes unterstützend tätig waren, herzlich dankt. 
